Kevin K. Kumar | Cody R. Goodwin | Michael A. Uhouse Julia Bornhorst | Tanja Schwerdtle | Michael Aschner | John A. McLean Aaron B. Bowman

\title{
Untargeted metabolic profiling identifies interactions between Huntington's disease and neuronal manganese status
}

Suggested citation referring to the original publication:

Metallomics 7 (2016), pp. 363-370

DOI http://dx.doi.org/10.1039/c4mt00223g

Postprint archived at the Institutional Repository of the Potsdam University in:

Postprints der Universität Potsdam

Mathematisch-Naturwissenschaftliche Reihe ; 232

ISSN 1866-8372

http://nbn-resolving.de/urn:nbn:de:kobv:517-opus4-94314 

CrossMark <click for updates

Cite this: Metallomics, 2015, 7,363

\section{Untargeted metabolic profiling identifies interactions between Huntington's disease and neuronal manganese status $\dagger$}

\author{
Kevin K. Kumar, $\ddagger^{\mathrm{a}}$ Cody R. Goodwin, $\ddagger^{\mathrm{b}}$ Michael A. Uhouse, ${ }^{a}$ Julia Bornhorst, $^{\mathrm{c}}$ \\ Tanja Schwerdtle, ${ }^{c}$ Michael Aschner, ${ }^{d}$ John A. McLean $\S^{\star b}$ and Aaron B. Bowman ${ }^{\star a}$
}

\begin{abstract}
Manganese $(\mathrm{Mn})$ is an essential micronutrient for development and function of the nervous system. Deficiencies in Mn transport have been implicated in the pathogenesis of Huntington's disease (HD), an autosomal dominant neurodegenerative disorder characterized by loss of medium spiny neurons of the striatum. Brain $\mathrm{Mn}$ levels are highest in striatum and other basal ganglia structures, the most sensitive brain regions to $\mathrm{Mn}$ neurotoxicity. Mouse models of $\mathrm{HD}$ exhibit decreased striatal $\mathrm{Mn}$ accumulation and HD striatal neuron models are resistant to Mn cytotoxicity. We hypothesized that the observed modulation of $\mathrm{Mn}$ cellular transport is associated with compensatory metabolic responses to HD pathology. Here we use an untargeted metabolomics approach by performing ultraperformance liquid chromatography-ion mobility-mass spectrometry (UPLC-IM-MS) on control and HD immortalized mouse striatal neurons to identify metabolic disruptions under three $\mathrm{Mn}$ exposure conditions, low (vehicle), moderate (non-cytotoxic) and high (cytotoxic). Our analysis revealed lower metabolite levels of pantothenic acid, and glutathione (GSH) in HD striatal cells relative to control cells. HD striatal cells also exhibited lower abundance and impaired induction of isobutyryl carnitine in response to increasing $\mathrm{Mn}$ exposure. In addition, we observed induction of metabolites in the pentose shunt pathway in HD striatal cells after high Mn exposure. These findings provide metabolic evidence of an interaction between the HD genotype and biologically relevant levels of $\mathrm{Mn}$ in a striatal cell model with known HD by Mn exposure interactions. The metabolic phenotypes detected support existing hypotheses that changes in energetic processes underlie the pathobiology of both HD and Mn neurotoxicity.
\end{abstract}

Received 31st August 2014, Accepted 8th January 2015

DOI: $10.1039 / \mathrm{c} 4 \mathrm{mt} 00223 \mathrm{~g}$

www.rsc.org/metallomics

\section{Introduction}

Huntington's disease (HD) is a highly debilitating, autosomaldominant neurodegenerative disorder characterized by motor dysfunction, behavioral abnormalities, and cognitive decline. ${ }^{1}$ In $\mathrm{HD}$, an expanded CAG repeat generates a pathogenic polyglutamine tract near the N-terminus of Huntingtin (HTT).

\footnotetext{
${ }^{a}$ Dept. of Neurology, Vanderbilt University, Nashville, TN, USA

${ }^{b}$ Dept. of Chemistry, Vanderbilt University, Nashville, TN, USA

${ }^{c}$ Institute of Nutritional Sciences, University of Potsdam, Nuthetal, Germany

${ }^{d}$ Dept. of Molecular Pharmacology, Albert Einstein College of Medicine, Bronx, NY, USA

$\dagger$ Electronic supplementary information (ESI) available. See DOI: 10.1039/ c4mt00223g

\# Both authors contributed equally to this manuscript.

$\S$ Deptartment of Chemistry, Vanderbilt University, 7330 Stevenson Center, Nashville, TN 37235, USA. E-mail: john.a.mclean@vanderbilt.edu; Fax: +1-615-343-1234; Tel: +1-615-322-1195.

T Deptartment of Neurology, Vanderbilt University Medical Center, 465 21st Avenue South, 6140 MRB3 Nashville, TN 37232-8552, USA. E-mail: aaron.bowman@vanderbilt.edu; Fax: +1-615-322-0486; Tel: +1-615-322-2651.
}

Expansion of this polyglutamine tract confers a toxic gain of function to HTT. ${ }^{2}$ Furthermore, though HTT is universally expressed, HD is characterized by selective neuropathological changes including atrophy of the caudate and putamen with prominent vulnerability of the striatal medium spiny neurons (MSNs). ${ }^{3}$

Although mechanisms of neurodegeneration in HD are still under investigation, several classes of neuronal stress have been implicated in its pathogenesis, including oxidative stress, mitochondrial dysfunction, glutamine sensitivity, and metabolic dysregulation. ${ }^{4}$ Congruently, many environmental toxicants are also known to elicit similar types of neuronal stress, such as the pro-oxidant metal manganese $(\mathrm{Mn})$, which causes striatal neurotoxicity in excess. ${ }^{5,6}$ MSNs in the striatum exhibit disruptions in neurochemistry and morphology in the context of $\mathrm{Mn}$ exposure. ${ }^{7}$ HD genotype prevents characteristic increases in MSN total dendritic length and branching found from week 13-16 in mouse postnatal development, and decreases in total spine density at week 16 of age. ${ }^{7}$ These pathophysiological changes in MSNs coincide with the onset hyperkinetic behavioral abnormalities observed in mouse models of HD. ${ }^{8}$ While the mechanism 
underlying disruptions in synaptic morphology that generate behavioral phenotypes is poorly understood, it has been proposed that downstream alterations in neuron connectivity and excitability impact signal propagation for higher functions. ${ }^{9-13}$ Mouse models of HD exhibit disruptions in dopaminergic signaling ${ }^{14-16}$ and levels of striatal dopamine. ${ }^{17}$ Similarly, neurotoxic exposures to Mn have been shown to deplete striatal dopamine levels. ${ }^{7} \mathrm{Mn}$ is regulated at a cell-level across neuronal differentiation ${ }^{18}$ and exposures to $\mathrm{Mn}$ have been associated with changes in energy metabolism..$^{19,20}$ Furthermore, both in vitro and in vivo mouse models expressing mutant HTT exhibit reduced total Mn accumulation. ${ }^{21}$ However, the mechanisms by which pathogenic alleles of HTT disrupt Mn transport are unknown. Here we test the hypothesis that the influence of HD genotype on Mn cellular transport processes is associated with compensatory metabolic responses to HD pathophysiological processes.

We interrogated the intracellular metabolite profiles from an immortalized striatal neuroprogenitor model of HD (mutant $S T H d h[\mathrm{Q} 111 / \mathrm{Q} 111]$ and wild type STHdh[Q7/Q7]) that is known to exhibit a strong HD-Mn interaction phenotype, ${ }^{21,22}$ under three extracellular $\mathrm{MnCl}_{2}$ exposure conditions (Fig. 1). Given that normal levels of brain $\mathrm{Mn}$ in mammals range from 20-53 $\mu \mathrm{M}$, we sought to assess the ability of Mn to influence the metabolic profile at low ( $0 \mu \mathrm{M}$ Mn added), moderate non-toxic (31 $\mu \mathrm{M} \mathrm{Mn})$, and high toxicological exposure concentrations $(125 \mu \mathrm{M} \mathrm{Mn}){ }^{23}$ These concentrations reflect over a 17 -fold change in cellular Mn levels, ranging from $\sim 5$ to $\sim 90 \mathrm{fmol} \mathrm{Mn} / 100$ cells with mutant cells accumulating less $\mathrm{Mn}$ than wild type cells consistent with our previous studies ${ }^{21,22}$ (Fig. 2). An untargeted assessment of intracellular metabolic perturbations was performed through a comprehensive small molecule profiling approach that integrates metabolomics, lipidomics, and glycomics, using ultra-performance liquid chromatography-ion mobility-mass spectrometry (UPLCIM-MS). ${ }^{24-30}$ This approach enabled study of HD-Mn interactions at a cellular level without a priori knowledge of HD or Mn

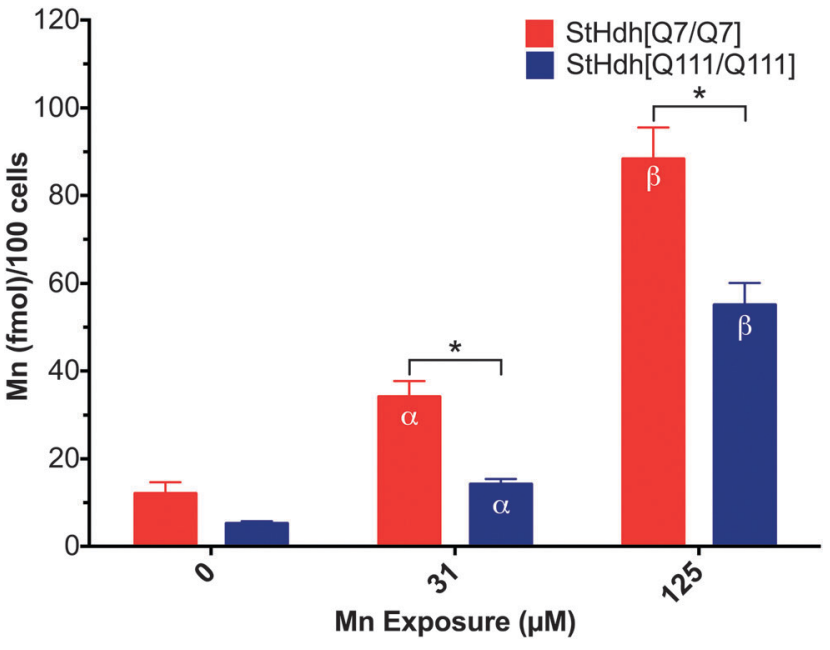

Fig. 2 Mn quantification using ICP-MS/MS. Plot of mean Mn (fmol)/100 cells for StHdh[Q7/Q7] and StHdh[Q111/111] across the three Mn exposure concentrations $(0,31,125 \mu \mathrm{M})$. Data are mean $\pm \operatorname{SEM}(n=5$ for StHdh[Q111/111] at $125 \mu \mathrm{M}, n=6$ for all other groups). Statistical significance evaluated by post hoc $t$-test with Bonferroni multi-testing correction. Label $(*)$ indicates significance $(p<0.01)$ for the genotype comparisons marked by brackets. Labels $(\alpha)$ and $(\beta)$ indicate significance $(p<0.01)$ comparing $(0$ vs. $31 \mu \mathrm{M})$ or (31 vs. $125 \mu \mathrm{M}$ ), respectively, within each genotype.

induced protein changes. Sampling the metabolite inventories of cells provides information representative of the striatal cell phenotype, and is reflective of the current biology of the system. The metabolomic profiling method has the potential to reveal mechanistic and therapeutic targets that may not be captured by genetic and transcriptional approaches. These present studies provide an integrated overview of the effects of genotype, Mn exposure, and HD-Mn interactions on metabolic pathways via quantifying their respective metabolites independent of any prior association with HD pathogenesis or Mn biology. Specifically, we investigated the metabolomic profiles of mutant and wild

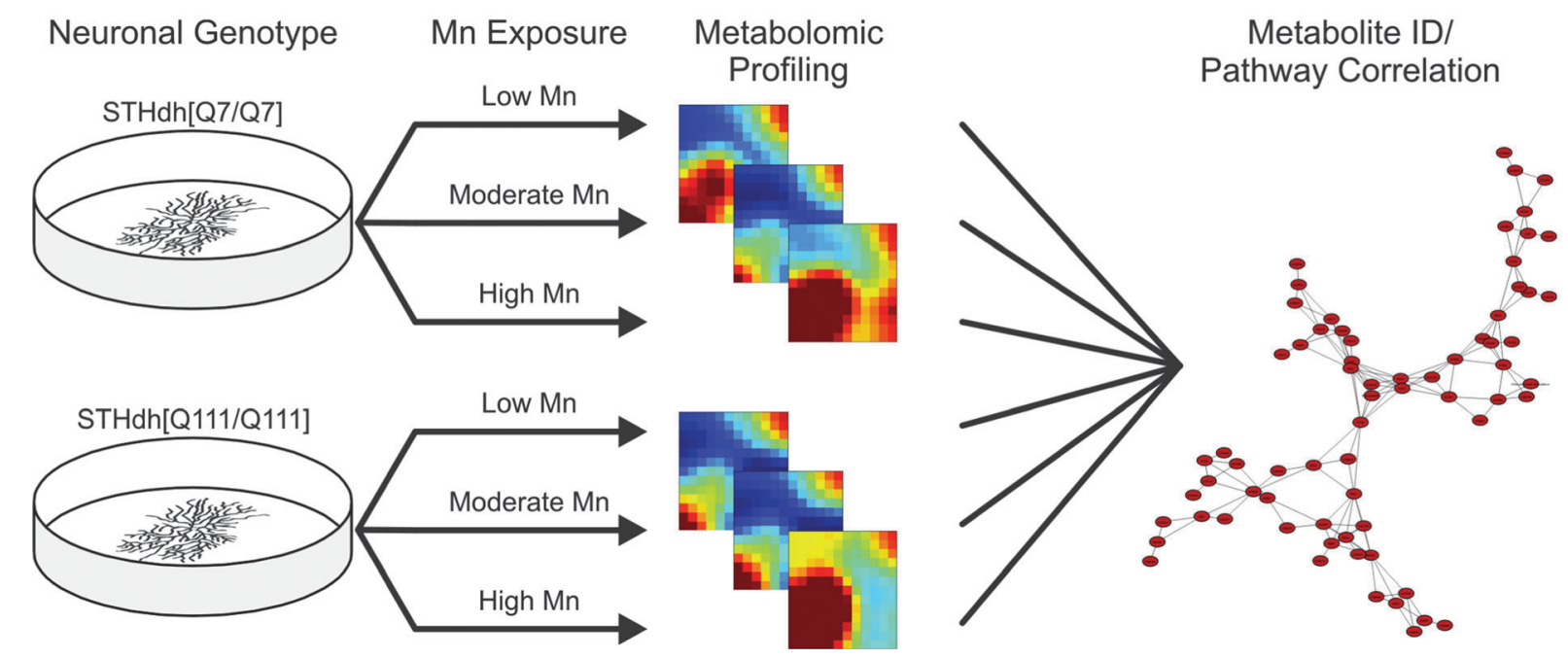

Fig. 1 Study of HD-Mn interaction using untargeted metabolomics. Schematic workflow for experimental approach. Mouse control (STHdh[Q7/Q7]) and HD mutant (STHdh[Q111/Q111]) striatal cells were treated with different Mn exposure conditions. Cells were harvested for metabolomics profiling and identification followed by pathway correlation. 
type immortalized striatal neuroprogenitors in response to increasing concentrations of extracellular $\mathrm{Mn} .{ }^{31}$ Basal metabolic differences in HD versus wild type striatal cells were observed, as well as significant differences in Mn-dependent and HD by Mn interactions in metabolite composition.

\section{Materials and methods}

\section{Cell culture and sample preparation}

Biological triplicates were prepared for all experiments performed. The clonal striatal cell lines-both mutant STHdh[Q111/Q111] and wild-type STHdh[Q7/Q7] were grown at $33{ }^{\circ} \mathrm{C}^{31}$ Culture and exposures were performed as previously described. ${ }^{21,32}$ Subsequently, STHdh[Q111/Q111] and wild-type STHdh[Q7/Q7] cells were plated at equal density 16 hours before treatment. Cells were exposed for 3 hours in Hank's Buffer Salt Solution (HBSS; Corning-CellGro) with or without $31 \mu \mathrm{M}$ or $125 \mu \mathrm{M} \mathrm{MnCl}_{2}$ added. Cells were harvested post-exposure by HPLC-grade methanol (Sigma Chemical Co, St. Louis, MO) extraction and snap frozen in liquid nitrogen. Prior to the analysis, samples were thawed and vigorously mixed and centrifuged at $4{ }^{\circ} \mathrm{C}$ and $14000 \times g$ for $10 \mathrm{~min}$ to precipitate proteins and particulates. The supernatants were transferred to a fresh tube and vacuum centrifuged until dry. The residuals were stored at $-80{ }^{\circ} \mathrm{C}$ until UPLC-IM-MS/MS sample preparation. For Mn quantification by ICP-MS/MS, the identical Mn exposure paradigm was utilized followed by harvesting in $1 \times$ PBS (Corning-CellGro) and centrifugation at $200 \times g$ for 5 minutes. The supernatant was aspirated and cell pellets were re-suspended in $550 \mu \mathrm{L}$ PBS with protease inhibitor cocktail (Sigma-Aldrich, St. Louis, MO). Samples were snap frozen in liquid nitrogen and stored at $-80{ }^{\circ} \mathrm{C}$ until analysis.

\section{Mn quantification using ICP-MS/MS}

An aliquot of each sample was taken for protein quantification using the Bradford Assay (Bio-Rad, Munich, Germany). Subsequently, Mn content was quantified using our previously published methods. ${ }^{33} \mathrm{Mn}$ (fmol)/100 cell count ratios were determined by quantified protein levels as previously reported. ${ }^{21,22}$ Total cellular $\mathrm{Mn}$ content is primarily reported on a per cell basis, as mutant STHdh[Q111/Q111] cells have $\sim 28 \%$ more protein than wild type STHdh[Q7/Q7] cells. ${ }^{22}$ Prism6 Graphpad Software was used for two-way ANOVA and post hoc $t$-test with Bonferroni multi-testing correction of binary group comparisons.

\section{UPLC-IM-MS/MS data acquisition}

The dried metabolite extracts were resuspended in $200 \mu \mathrm{L}$ water with $0.1 \%$ formic acid. UPLC-IM-MS/MS (Synapt G2 HDMS, Waters Corp., Milford, MS) data acquisition was performed by continuous fragmentation of all species using a ramped collision-induced dissociation (CID) energy program and correlation of the fragment to precursor ions through alignment of chromatography traces and ion mobility drift times termed $\mathrm{MS}^{\mathrm{E}}$. The UPLC was performed with a 30 minute gradient. Mobile phase A consisted of $\mathrm{H}_{2} \mathrm{O}$ with $0.1 \%$ formic acid and mobile phase B consisted of ACN with $0.1 \%$ formic acid.
A $1 \times 100 \mathrm{~mm} 1.7 \mu \mathrm{m}$ particle BEH-T3 $\mathrm{C}_{18}$ column (Waters Corp.) was used for chromatographic separations with a flow rate of $75 \mu \mathrm{L} \mathrm{min}{ }^{-1}$, a column temperature of $40{ }^{\circ} \mathrm{C}$. An autosampler was used for sample injection and held at $4{ }^{\circ} \mathrm{C}$, with a loop size of $5 \mu \mathrm{L}$. The initial solvent composition was $100 \%$ A, which was held for $1 \mathrm{~min}$ and ramped to $0 \%$ A over the next 11 minutes, held at $0 \%$ A for 2 minutes and returned to $100 \%$ A over a 0.1 minute period. The gradient was held at $100 \% \mathrm{~A}$ for the next 10.9 minutes for equilibration. Ten column-load injections were performed with $5 \mu \mathrm{L}$ injections of the quality control. Quality control injections were then performed after every 10th sample injection to ensure instrument stability.

Continuous IM-MS ${ }^{\mathrm{E}}$ spectra were acquired at a rate of $2 \mathrm{~Hz}$ from 50-2000 Da in positive mode for the duration of the injection. The instrument was calibrated to less than 1 ppm mass accuracy using sodium formate clusters prior to analysis. A two-point internal standard of leucine enkephalin was infused in parallel at a flow rate of $7 \mu \mathrm{L} \mathrm{min}{ }^{-1}$ and acquired every 10 seconds. The source capillary was held at $110{ }^{\circ} \mathrm{C}$ and $3.0 \mathrm{kV}$, with a desolvation gas flow of $400 \mathrm{~L} \mathrm{~h}^{-1}$ and a temperature of $150{ }^{\circ} \mathrm{C}$. The sampling cone was held at a setting of 35.0, with the extraction cone at a setting of 5.0. In the $\mathrm{MS}^{\mathrm{E}}$ configuration, low- and high-energy spectra are acquired for each scan. High energy data performed a collision energy profile from $10-30 \mathrm{eV}$ in the trapping region, providing postmobility fragmentation. Ion mobility separations were performed with a wave velocity of $550 \mathrm{~m} \mathrm{~s}^{-1}$, a wave height of $40.0 \mathrm{~V}$, and a

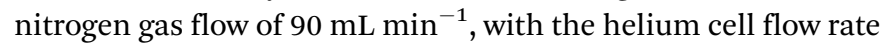
at $180 \mathrm{~mL} \mathrm{~min}{ }^{-1}$.

\section{Data processing and statistical analysis}

Data were converted to mzXML format using the msconvert tool from the ProteoWizard package as previously described. ${ }^{34}$ Peak picking and alignment were performed using XCMS in $\mathrm{R}^{35}$ The resulting data matrix contained 475 detected features. Features were pre-filtered for reproducibility using an ANOVA threshold of $p \leq 0.10$, comparing across all experimental conditions and biological replicates. Prior to self-organizing map (SOM) analyses, MVSA, and further statistical analyses, analytical triplicates were averaged. For Molecular Expression Dynamics Investigator (MEDI) analysis, ${ }^{29}$ a grid of $25 \times 26$ was generated, with 100 first phase training iterations and 160 second phase. An initial training radius of 10.0 was defined with a learning factor of 0.5 , a neighborhood block size of 20, and a conscience of 5.0. For the second phase, a neighborhood radius of 1.0, learning factor of 0.05 , neighborhood block size of 2 , and conscience of 2.0 was defined. A random seed of 10 with a Pearson's correlation distance metric and random selection initialization was used. Further statistical analyses were performed in SPSS version 22 (SPSS, Inc., Chicago, IL).

\section{Metabolite identification}

Metabolite identifications were performed using accurate mass measurements and fragmentation spectra extracted from IM-MS ${ }^{\mathrm{E}}$ data. Utilizing drift time correlations, product ions were correlated appropriately to precursors for extraction of high-energy spectra. Annotated spectra may be found in the ESI $\dagger$ (Fig. S1-S5). 
Identification confidence levels are in accordance with the Metabolomics Standards Initiative proposed minimum reporting standards. ${ }^{36}$ Metabolites were prioritized using the SOM methodology and five were subsequently identified. These putative identifications are supported by accurate mass measurements and fragmentation data interpretation aided through database searching (Table S1, ESI $\dagger$ ). Fragmentation data were acquired using the untargeted fragmentation of all ions postmobility separation, which were subsequently isolated using mobility correlations Metlin, ${ }^{37}$ the Human Metabolome Database $^{38,39}$ and LIPID MAPS. ${ }^{40}$

\section{Results}

We quantified total cellular Mn in both wild type STHdh[Q7/Q7] and mutant STHdh[Q111/Q111] cells following the 3 hour $\mathrm{MnCl}_{2}$ exposure in HBSS (Fig. 2). Wild type STHdh[Q7/Q7] cells had total cellular Mn levels of 12, 34 and $88 \mathrm{fmol} \mathrm{Mn/100} \mathrm{cells}$ (equivalent to $0.032,0.090$ and $0.234 \mathrm{ng} \mathrm{Mn}$ per $\mu \mathrm{g}$ protein). Mutant STHdh[Q111/Q111] cells had total cellular Mn levels of 5.2, 14 and $55 \mathrm{fmol} \mathrm{Mn/100} \mathrm{cells} \mathrm{(equivalent} \mathrm{to} \mathrm{0.018,} 0.048$ and $0.187 \mathrm{ng}$ Mn per $\mu \mathrm{g}$ protein). Two-way ANOVA found a significant effect by genotype $(p<0.001)$, Mn exposure $(p<0.001)$ and a genotype by $\mathrm{Mn}$ interaction effect $(p=0.01)$. The wild type STHdh[Q7/Q7] cells had significantly more total Mn content versus mutant STHdh[Q111/Q111] cells at both $31 \mu \mathrm{M}$ and $125 \mu \mathrm{M} \mathrm{MnCl} \mathrm{M}_{2}$ exposures $(p<0.01 t$-test with Bonferroni correction) and trended higher under the no added Mn exposure ( $p=0.02 t$-test without multi-testing correction).

In all, 475 features were detected with unique retention time and $\mathrm{m} / \mathrm{z}$ values. To determine peak stability, we performed ANOVA across all samples, grouping technical replicates and removing all values with a $p$-value greater than 0.10 . This resulted in 362 filtered features for all subsequent analyses. Our data suggest deviations in the metabolomic profiles of both control and HD striatal cell lines are driven primarily by the concentration of Mn to which they are exposed. Principle component analysis (PCA) shows separation of global metabolomic profiles in principal component one (PC1) based upon treatment conditions (Fig. 3). Each marker represents the average metabolomic profile of one biological sample analyzed in triplicate and ANOVA filtered. Biological replicates cluster in PCA space, indicating biological consistency of these measurements. However, PC2 separates metabolomic profiles predominantly based upon cell genotype, suggesting differences in basal metabolism between the metabolomic profiles of STHdh[Q7/Q7] and STHdh[Q111/Q111] cells. Self-organized heat maps (Fig. 4) that cluster metabolites by covariance were then used to visualize global patterns of metabolite changes between control and HD striatal cells with varying $\mathrm{Mn}$ exposures. ${ }^{29,41}$ In this method, metabolites self-assemble into groups in a user-defined grid based upon similarities in abundance profiles using an unsupervised computational process. For example, metabolites that are increased in abundance consistently across biological replicates as a generic response to $\mathrm{Mn}$ concentration will be grouped in a region of the

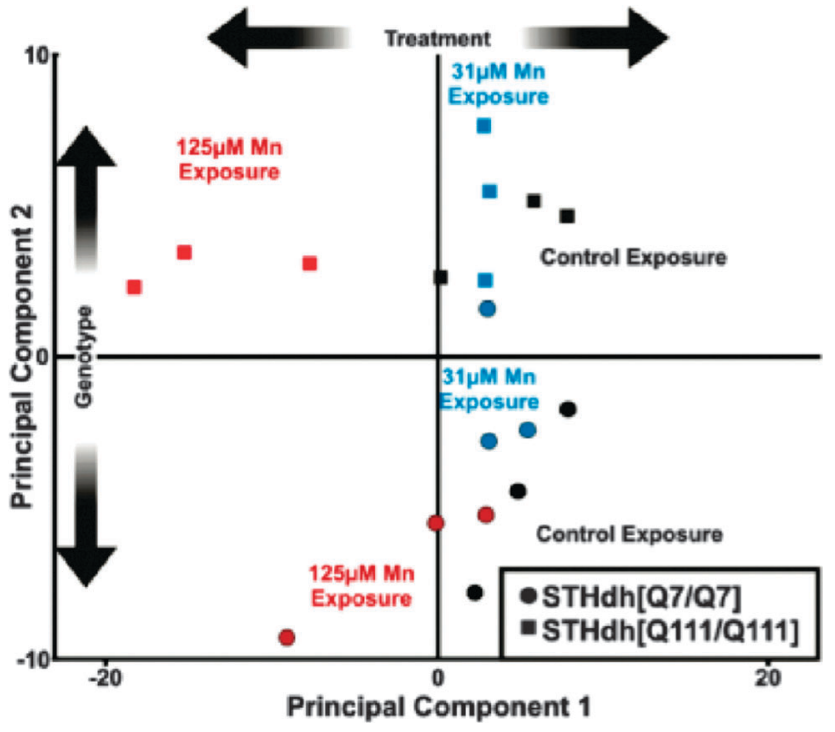

Fig. 3 Principal component analysis of metabolomic profiles. Plots of principal component analyses for STHdh[Q7/Q7] and STHdh[Q111/Q111] cells exposed to varying physiologically-relevant $\mathrm{Mn}$ concentrations.

SOM space, while metabolites that are produced as a genotypespecific response to Mn concentration will group in a separate, distinct region of SOM space. Experimental groups are then represented as heat maps based upon the abundances of organized metabolites. The number of metabolites seeded in each grid location of the SOM (Fig. 4) is indicated in the metabolite density map, with blue representing one metabolite and red corresponding to 44 metabolites. The largest metabolite abundance occupying the central region of the SOM space corresponded to a small protein with a molecular weight of 8561.6 Da, putatively identified as ubiquitin based upon accurate mass (Fig. S3, ESI $\dagger$ ). After SOM prioritization and analysis, we observed a decreased production of glutathione (GSH) and pantothenic acid in the STHdh[Q111/Q111] cell line. A general linear model was applied to evaluate significant differences in the abundance of metabolites by genotype, $\mathrm{Mn}$ exposure, or genotype by Mn exposure interaction (Fig. 5A-D). A significant relationship between genotype and both GSH and pantothenic acid abundance was observed $(p<0.001)$. Both genotype $(p<0.05)$ and Mn exposure $(p<0.005)$ had a significant effect on the levels of isobutyryl carnitine. There was also a statistically significant genotype by Mn exposure interaction effect $(p<0.05)$ on isobutyryl carnitine abundance. Finally, ribulose-5-phosphate, showed a significant change in abundance by Mn exposure $(p<0.001)$, genotype $(p<0.001)$, and a genotype by $\mathrm{Mn}$ exposure interaction effect $(p<0.01)$.

\section{Discussion}

The novel application of untargeted metabolomic profiling to characterize Mn-dependent cellular responses in an HD striatal cell model highlighted specific alterations in metabolism. These findings support a hypothesis of altered cellular energetics by 


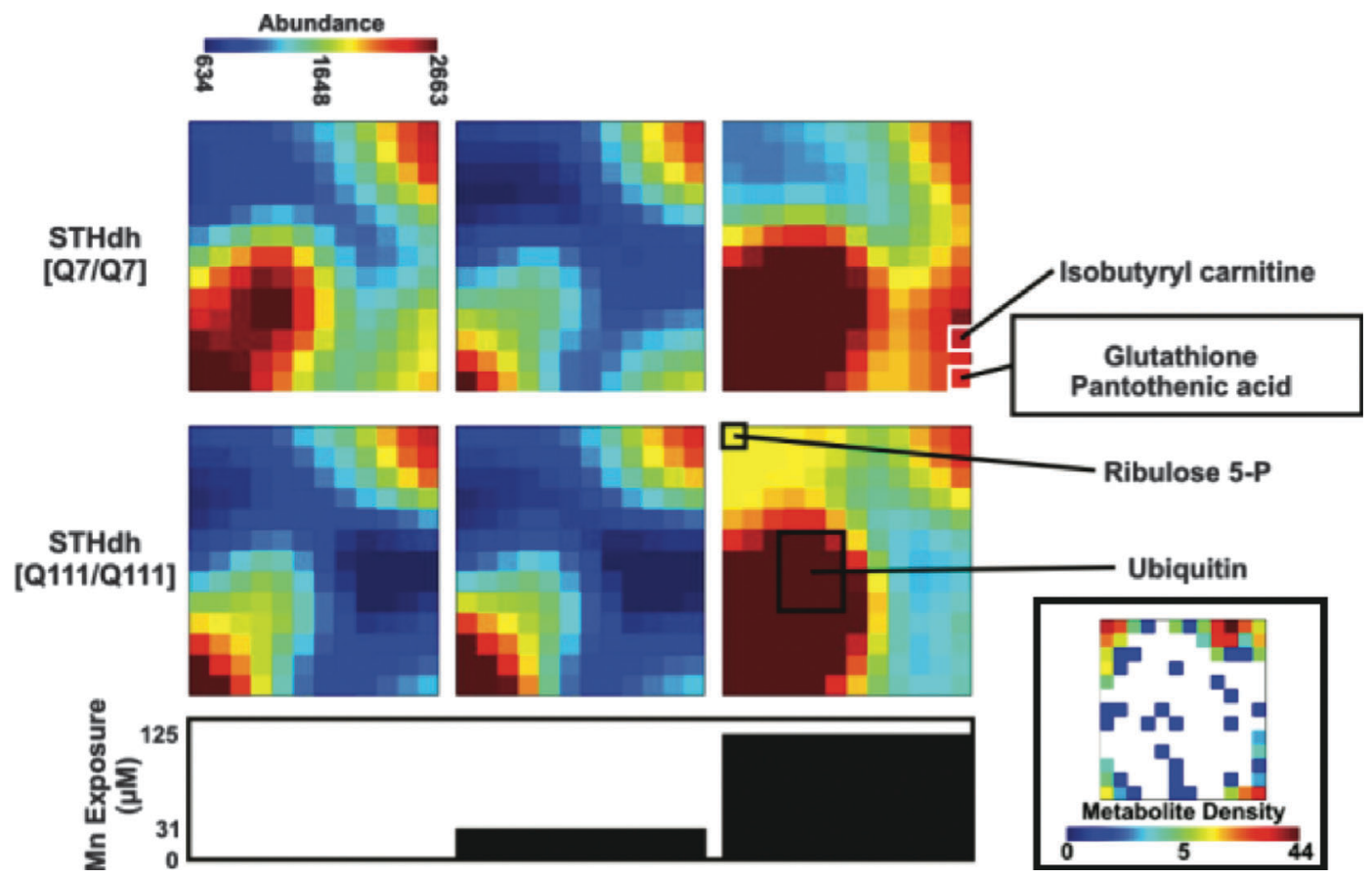

Fig. 4 Self-organized metabolic heat maps with prioritized metabolites. Annotated self-organized heat maps are shown with scales for identified metabolite abundance and metabolite density by map region.
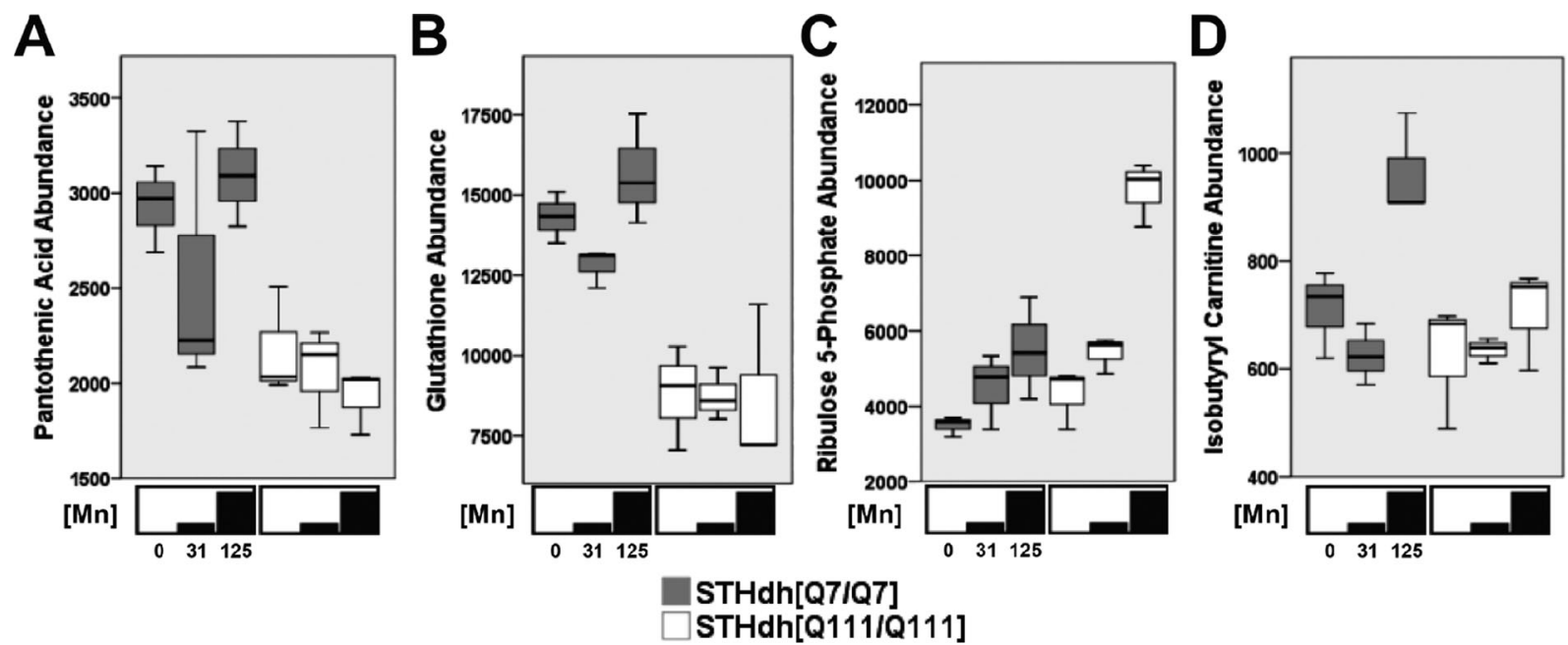

Fig. 5 Box plot production profiles for significant metabolites. Box plot production profiles for (A) pantothenic acid, (B) glutathione, (C) ribulose 5-phosphate, and (D) isobutyryl carnitine are shown. STHdh[Q7/Q7] is represented by gray bars, STHdh[Q111/Q111] represented by white. Mn concentration is indicated on abscissa with standard error about the mean. Mn exposure concentrations are indicated by black bars below each panel.

pathogenic alleles of HTT, varying Mn exposure conditions, and well as HD by Mn interaction effects.

\section{HD striatal cells exhibit lower levels of pantothenic acid and GSH}

The identification of lower levels of pantothenic acid (vitamin B5) and GSH in HD striatal cells fits into the complex biology mediating neuronal mitochondrial processes and metal handling. Notably, disruptions in pantothenic acid levels have been described in a related neurodegenerative disorder, neurodegeneration with brain iron accumulation (NBIA). ${ }^{42,43}$ Mutations in pantothenate kinase 2 (PANK2), encoding a neuronal mitochondrial protein responsible for catalyzing the phosphorylation of pantothenic acid to phosphopantothenate, have been identified in one-third of patients with this disorder. ${ }^{44,45}$ This process is critical to the synthesis of coenzyme (CoA), a universal acyl carrier involved in the citric acid cycle and cellular energetics. In addition, pantothenic acid is a necessary co-enzyme for the synthesis of $\mathrm{GSH}$, and has been shown to protect cells from oxidative damage by increasing free GSH levels. ${ }^{46}$ Given our observation of 
decreased pantothenic acid levels relative to control, downstream impaired cellular energetics and exhausted response to oxidative stress could play a role in neurodegeneration observed in HD. Mn neurotoxicity can result from enhanced production of free radicals that are scavenged by GSH and other antioxidants. ${ }^{47}$ However, the decreased abundance of GSH and pantothenic acid we observed appear to be independent of cellular Mn burden given the lack of significant Mn-dependent changes. This finding is supported by previously reported reductions in levels of glutathione disulfide from brains of patients with HD. ${ }^{48}$ Our data suggest HD genotype-dependent changes in GSH and pantothenic acid may be upstream or independent of Mn-dependent changes in cellular biology.

\section{Alterations in levels of ribulose-5 phosphate implicate modulation of the pentose shunt pathway in HD}

The pentose shunt pathway functions to regenerate the reducing agent NADPH, which is necessary for biosynthetic processes in the brain, such as synthesis of free fatty acids from acetyl-CoA. ${ }^{49}$ The dehydrogenation of glucose 6-phosphate to ribulose 5-phosphate is the rate-limiting step of the pentose shunt pathway. Furthermore, the pentose shunt pathway is induced by p53, a protein known to mediate cellular dysfunction and behavioral abnormalities in HD. ${ }^{50}$ Our data suggest increasing cellular $\mathrm{Mn}$ burden results in a corresponding induction of ribulose 5-phosphate, an effect that was exaggerated in HD striatal cells relative to control. This finding corroborates previous studies that demonstrate HD striatal cell impairments in mitochondrial dynamics and energetics. ${ }^{51,52}$ The genotype-dependent difference was apparent only at the cytotoxic $125 \mu \mathrm{M}$ Mn exposure, while increasing cellular Mn burden from vehicle to $31 \mu \mathrm{M}$ does not significantly increase ribulose 5-phosphate levels. This is consistent with an involvement of ribulose 5-phosphate metabolism in the Mn cytotoxic cellular response. Indeed, NADPH generated in production of ribulose 5 -phosphate mediates the generation of GSH by reducing glutathione disulfide. Increased ribulose 5-phosphate levels suggest that HD striatal cells are hypersensitive to Mn related oxidative stress, despite their reduced net $\mathrm{Mn}$ accumulation. In addition, when taken into the context of decreased levels of glutathione and pantothenic acid in HD striatal cells, increased levels of ribulose 5-phosphate may be a cellular response which increases the reduced amount of GSH available for handling oxidative stress.

\section{HD striatal cells exhibit lower abundance and an impaired Mn-dependent increase of isobutyryl carnitine}

Isobutyryl carnitine is a product of the acyl-CoA dehydrogenases, mitochondrial enzymes that are involved in the process of metabolism of fatty acids or branched-chain amino acids. In addition to these functions, carnitines have well described roles in the brain including: lipid synthesis, membrane composition, expression modulation, mitochondrial energetics, activation of antioxidant mechanisms, and strengthening cholinergic neurotransmission. ${ }^{53}$ Although, increases in isobutyryl carnitine levels have been observed in inborn errors of metabolism such as short chain acyl dehydrogenase deficiency, reduced levels of this metabolite have not been reported in the context of any neurodegenerative disease. ${ }^{54}$ Our data reveal genotype, Mn exposure and genotype by $\mathrm{Mn}$ interaction effects for levels of isobutyryl carnitine. We observed that $\mathrm{Mn}$ exposure induces elevated levels of isobutyryl carnitine in control striatal cells but not the HD mutant cells. As HD striatal cells accumulate significantly less cellular Mn levels versus control cells, ${ }^{12,26}$ the genotype specific effect is likely due to a failure to reach a sufficient level of intracellular Mn. The observation that levels of isobutyryl carnitine increased in control striatal cells only at the highest Mn exposure is consistent with this interpretation. Mn toxicity occurs in part via impairment of mitochondrial function. ${ }^{55,56}$ The increase in this metabolite under conditions of heavy $\mathrm{Mn}$ burden may reflect increased reliance on isobutyryl carnitine-mediated process to generate acetyl-CoA for ATP synthesis.

\section{Modeling the HD-Mn striatal cell metabolomics}

The striatum is a unique region of the brain, linked to both neurodegeneration in HD and Mn neurotoxicity. The application of untargeted metabolomics to elucidate the mechanisms through which control and HD mouse striatal cells respond to low, moderate non-toxic, and high toxicological Mn levels revealed selective alterations in cellular stress responses. Given that the HD genotype disrupts a variety of cellular processes including transcriptional regulation, HD striatal cells may have adapted their metabolism to survive under this genotypic stressor. Their response to the pathogenic processes of mutant HTT may activate compensatory processes that influence Mn toxicity and oxidative stress metabolites (Fig. 6). In modeling the changes we observe, we postulate that the HD genotype produces a state of chronic oxidative stress, depleting cellular $\mathrm{GSH}$ reserves. In response, HD striatal cells have a deficit in pantothenic acid, hampering efforts to replenish the limited GSH levels. A compensatory process may be induced by HD striatal cells, increased reliance on the pentose shunt pathway

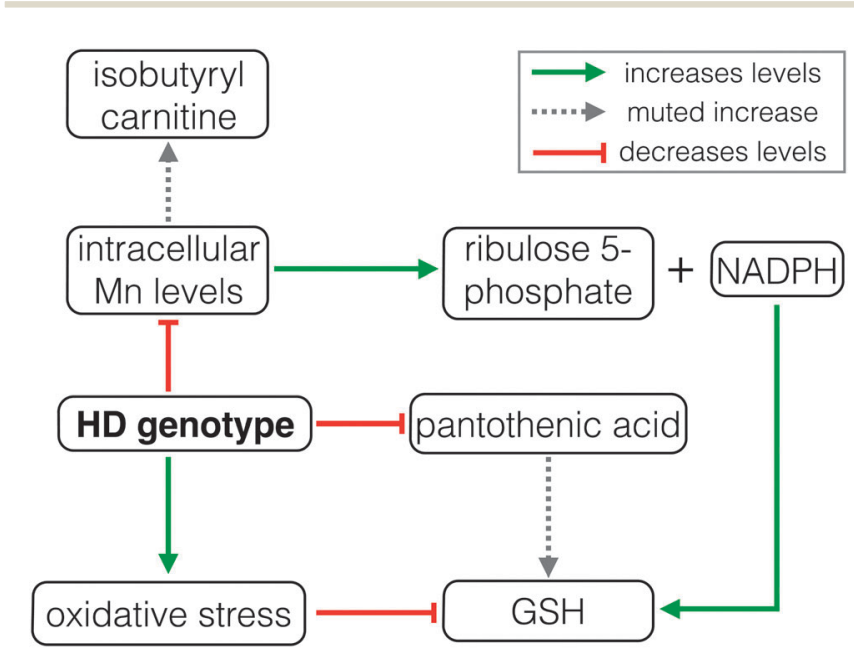

Fig. 6 Metabolic disruption in Huntington's disease. Summary diagram describing the proposed model of HD-Mn interaction based on untargeted metabolomics. Increased metabolite levels indicated by arrows (green), decreased metabolite levels by lines (red), and muted increases in metabolites by dashed arrows (gray). 
to regenerate GSH, when cellular Mn burden reaches cytotoxic levels. The exaggerated response to $\mathrm{Mn}$ is especially noteworthy given the decreased ability of HD striatal cells to accumulate Mn. ${ }^{21,22}$ The decreased Mn uptake by HD striatal cells may also explain the selective increase of isobutyryl carnitine levels in control cells at toxic Mn levels.

\section{Conclusion}

In summary, we report the application of untargeted metabolomics to provide insight into the processes mediating the complex interactions of genotype and levels of the essential metal, $\mathrm{Mn}$, in the context of HD. This unbiased approach revealed disruption in the levels of several diverse physiological and energetic processes by $\mathrm{Mn}$ and/or HD genotype. Furthermore, the examination of response to low, moderate, and high Mn levels using untargeted metabolomics provided a unique perspective on the response of HD striatal cells on a global cellular scale. While we identified metabolites with known links to $\mathrm{HD}$ pathogenesis such as $\mathrm{GSH}^{57}$ the classification of changes in pantothenic acid and the pentose shunt pathway implicate disruption in basal energetic metabolism in HD. In addition, our studies identify alterations in the isobutyryl carnitine, a product of fatty acid metabolism. Further examination of the identified cellular processes may provide future mechanistic understanding into the loss of striatal neurons in HD and may serve as a framework for future therapeutic interventions for this highly debilitating disorder. Additionally, our successful identification of metabolites implicated in HD pathogenesis provides evidence for the value of untargeted metabolomics using SOM-based MEDI analysis. Future application of this method to other systems provides an opportunity for chemists to gain insight into biological processes previously intractable to study by conventional molecular and cell biology techniques. Our approach can be applied to a variety of other poorly understood disease contexts where a particular environmental exposure is linked to disease progression or severity.

\section{Acknowledgements}

K.K.K. acknowledges support by Public Health Service award T32 GM07347 from the National Institute of General Medical Studies for the Vanderbilt Medical-Scientist Training Program. A.B.B and M.A acknowledge support by the NIH National Institute of Environmental Health Sciences R01 ES016931 (A.B.B.) and ES010563 (A.B.B. and M.A.). C.R.G. and J.A.M. acknowledge support by the NIH National Center for Advancing Translational Sciences (UH2TR000491); the Defense Threat Reduction Agency (HDTRA1-09-1-00-13); the Vanderbilt Institute of Chemical Biology; and the Vanderbilt Institute for Integrative Biosystems Research and Education.

\section{References}

1 F. Walker, Lancet, 2007, 369, 218-228.

2 F. Giorgini, Proc. Natl. Acad. Sci. U. S. A., 2013, 110, 14516-14517.
3 J. P. Vonsattel and M. DiFiglia, J. Neuropathol. Exp. Neurol., 1998, 57, 369-384.

4 M. T. Lin and F. M. Beal, Nature, 2006, 443, 787-795.

5 X. Liu, K. A. Sullivan, J. E. Madl, M. Legare and R. B. Tjalkens, Toxicol. Sci., 2006, 91, 521-531.

6 E. P. Brouillet, L. Shinobu, U. McGarvey, F. Hochberg and M. F. Beal, Exp. Neurol., 1993, 120, 89-94.

7 J. L. Madison, M. Wegrzynowicz, M. Aschner and A. B. Bowman, PLoS One, 2012, 7, e31024.

8 E. J. Slow, J. van Raamsdonk, D. Rogers, S. H. Coleman, R. K. Graham, Y. Deng, R. Oh, N. Bissada, S. M. Hossain, Y. Z. Yang, X. J. Li, E. M. Simpson, C. A. Gutekunst, B. R. Leavitt and M. R. Hayden, Hum. Mol. Genet., 2003, 12, 1555-1567.

9 C. Schmidt-Hieber, P. Jonas and J. Bischofberger, J. Neurosci., 2007, 27, 8430-8441.

10 Z. F. Mainen and T. J. Sejnowski, Nature, 1996, 382, 363-366. 11 D. Tsay and R. Yuste, Trends Neurosci., 2004, 27, 77-83.

12 S. W. Jaslove, Neuroscience, 1992, 47, 495-519.

13 C. S. Woolley, N. G. Weiland, B. S. McEwen and P. A. Schwartzkroin, J. Neurosci., 1997, 17, 1848-1859.

14 A. N. Ortiz, B. J. Kurth, G. L. Osterhaus and M. A. Johnson, Neurosci. Lett., 2011, 492, 11-14.

15 J. H. Cha, C. M. Kosinski, J. A. Kerner, S. A. Alsdorf, L. Mangiarini, S. W. Davies, J. B. Penney, G. P. Bates and A. B. Young, Proc. Natl. Acad. Sci. U. S. A., 1998, 95, 6480-6485. 16 J. A. Bibb, Z. Yan, P. Svenningsson, G. L. Snyder, V. A. Pieribone, A. Horiuchi, A. C. Nairn, A. Messer and P. Greengard, Proc. Natl. Acad. Sci. U. S. A., 2000, 97, 6809-6814.

17 T. S. Tang, X. Chen, J. Liu and I. Bezprozvanny, J. Neurosci., 2007, 27, 7899-7910.

18 K. K. Kumar, E. W. Lowe, Jr., A. A. Aboud, M. D. Neely, R. Redha, J. A. Bauer, M. Odak, C. D. Weaver, J. Meiler, M. Aschner and A. B. Bowman, Sci. Rep., 2014, 4, 6801.

19 T. R. Guilarte, Neurotoxicology, 2010, 31, 572-574.

20 J. Levin, U. Bertsch, H. Kretzschmar and A. Giese, Biochem. Biophys. Res. Commun., 2005, 329, 1200-1207.

21 B. B. Williams, D. Li, M. Wegrzynowicz, B. K. Vadodaria, J. G. Anderson, G. F. Kwakye, M. Aschner, K. M. Erikson and A. B. Bowman, J. Neurochem., 2010, 112, 227-237.

22 B. B. Williams, G. F. Kwakye, M. Wegrzynowicz, D. Li, M. Aschner, K. M. Erikson and A. B. Bowman, Toxicol. Sci., 2010, 117, 169-179.

23 A. B. Bowman and M. Aschner, Neurotoxicology, 2014, 41, 141-142.

24 D. K. Derewacz, C. R. Goodwin, C. R. McNees, J. A. McLean and B. O. Bachmann, Proc. Natl. Acad. Sci. U. S. A., 2013, 110, 2336-2341.

25 J. C. May, C. R. Goodwin, N. M. Lareau, K. L. Leaptrot, C. B. Morris, R. T. Kurulugama, A. Mordehai, C. Klein, W. Barry, E. Darland, G. Overney, K. Imatani, G. C. Stafford, J. C. Fjeldsted and J. A. McLean, Anal. Chem., 2014, 86, 2107-2116.

26 L. S. Fenn, M. Kliman, A. Mahsut, S. R. Zhao and J. A. McLean, Anal. Bioanal. Chem., 2009, 394, 235-244.

27 J. A. McLean, J. Am. Soc. Mass Spectrom., 2009, 20, 1775-1781. 
28 L. S. Fenn and J. A. McLean, Anal. Bioanal. Chem., 2008, 391, 905-909.

29 C. R. Goodwin, S. D. Sherrod, C. C. Marasco, B. O. Bachmann, N. Schramm-Sapyta, J. P. Wikswo and J. A. McLean, Anal. Chem., 2014, 86, 6563-6571.

30 K. M. Hines, S. Ashfaq, J. M. Davidson, S. R. Opalenik, J. P. Wikswo and J. A. McLean, Anal. Chem., 2013, 85, 3651-3659.

31 F. Trettel, D. Rigamonti, P. Hilditch-Maguire, V. C. Wheeler, A. H. Sharp, F. Persichetti, E. Cattaneo and M. E. MacDonald, Hum. Mol. Genet., 2000, 9, 2799-2809.

32 K. K. Kumar, A. A. Aboud, D. K. Patel, M. Aschner and A. B. Bowman, J. Biochem. Mol. Toxicol., 2013, 27, 42-49.

33 J. Bornhorst, S. Chakraborty, S. Meyer, H. Lohren, S. G. Brinkhaus, A. L. Knight, K. A. Caldwell, G. A. Caldwell, U. Karst, T. Schwerdtle, A. Bowman and M. Aschner, Metallomics, 2014, 6, 476-490.

34 D. Kessner, M. Chambers, R. Burke, D. Agus and P. Mallick, Bioinformatics, 2008, 24, 2534-2536.

35 C. A. Smith, J. Elizabeth, G. O'Maille, R. Abagyan and G. Siuzdak, Anal. Chem., 2006, 78, 779-787.

36 L. W. Sumner, A. Amberg, D. Barrett, M. H. Beale, R. Beger, C. A. Daykin, T. W. M. Fan, O. Fiehn, R. Goodacre and J. L. Griffin, Metabolomics, 2007, 3, 211-221.

37 C. A. Smith, G. O’Maille, E. J. Want, C. Qin, S. A. Trauger, T. R. Brandon, D. E. Custodio, R. Abagyan and G. Siuzdak, Ther. Drug Monit., 2005, 27, 747-751.

38 D. S. Wishart, C. Knox, A. C. Guo, R. Eisner, N. Young, B. Gautam, D. D. Hau, N. Psychogios, E. Dong, S. Bouatra, R. Mandal, I. Sinelnikov, J. Xia, L. Jia, J. A. Cruz, E. Lim, C. A. Sobsey, S. Shrivastava, P. Huang, P. Liu, L. Fang, J. Peng, R. Fradette, D. Cheng, D. Tzur, M. Clements, A. Lewis, A. De Souza, A. Zuniga, M. Dawe, Y. Xiong, D. Clive, R. Greiner, A. Nazyrova, R. Shaykhutdinov, L. Li, H. J. Vogel and I. Forsythe, Nucleic Acids Res., 2009, 37, D603-D610.

39 D. S. Wishart, D. Tzur, C. Knox, R. Eisner, A. C. Guo, N. Young, D. Cheng, K. Jewell, D. Arndt, S. Sawhney, C. Fung, L. Nikolai, M. Lewis, M. A. Coutouly, I. Forsythe, P. Tang, S. Shrivastava, K. Jeroncic, P. Stothard, G. Amegbey, D. Block, D. D. Hau, J. Wagner, J. Miniaci, M. Clements, M. Gebremedhin, N. Guo, Y. Zhang, G. E. Duggan, G. D. Macinnis, A. M. Weljie, R. Dowlatabadi, F. Bamforth, D. Clive, R. Greiner, L. Li, T. Marrie, B. D. Sykes, H. J. Vogel and L. Querengesser, Nucleic Acids Res., 2007, 35, D521-D526.
40 M. Sud, E. Fahy, D. Cotter, A. Brown, E. A. Dennis, C. K. Glass, A. H. Merrill, Jr., R. C. Murphy, C. R. Raetz, D. W. Russell and S. Subramaniam, Nucleic Acids Res., 2007, 35, D527-D532.

41 G. S. Eichler, S. Huang and D. E. Ingber, Bioinformatics, 2003, 19, 2321-2322.

42 P. T. Kotzbauer, A. C. Truax, J. Q. Trojanowski and V. M. Lee, J. Neurosci., 2005, 25, 689-698.

43 K. F. Swaiman, Arch. Neurol., 1991, 48, 1285-1293.

44 T. P. Begley, C. Kinsland and E. Strauss, Vitam. Horm., 2001, 61, 157-171.

45 S. J. Hayflick, S. K. Westaway, B. Levinson, B. Zhou, M. A. Johnson, K. H. Ching and J. Gitschier, N. Engl. J. Med., 2003, 348, 33-40.

46 V. S. Slyshenkov, D. Dymkowska and L. Wojtczak, FEBS Lett., 2004, 569, 169-172.

47 A. Vescovi, M. Gebbia, G. Cappelletti, E. A. Parati and A. Santagostino, Toxicology, 1989, 57, 183-191.

48 J. Sian, D. T. Dexter, A. J. Lees, S. Daniel, Y. Agid, F. JavoyAgid, P. Jenner and C. D. Marsden, Ann. Neurol., 1994, 36, 348-355.

49 P. J. Magistretti and I. Allaman, Brain energy metabolism, Springer, 2013, pp. 1591-1620.

50 B. I. Bae, H. Xu, S. Igarashi, M. Fujimuro, N. Agrawal, Y. Taya, S. D. Hayward, T. H. Moran, C. Montell, C. A. Ross, S. H. Snyder and A. Sawa, Neuron, 2005, 47, 29-41.

51 Y. N. Jin, W. Y. Hwang, C. Jo and G. V. Johnson, PLoS One, 2011, 7, e30406.

52 Y. N. Jin, Y. V. Yu, S. Gundemir, C. Jo, M. Cui, K. Tieu and G. V. Johnson, PLoS One, 2012, 8, e57932.

53 L. L. Jones, D. A. McDonald and P. R. Borum, Prog. Lipid Res., 2010, 49, 61-75.

54 D. D. Koeberl, S. P. Young, N. S. Gregersen, J. Vockley, W. E. Smith, D. K. Benjamin Jr, Y. An, S. D. Weavil, S. H. Chaing, D. Bali, M. T. McDonald, P. S. Kishnani, Y. T. Chen and D. S. Millington, Pediatr. Res., 2003, 54, 219-223; C. Zwingmann, D. Leibfritz and A. S. Hazell, Neurotoxicology, 2004, 25, 573-587.

55 C. Zwingmann, D. Leibfritz and A. S. Hazell, Neurotoxicology, 2004, 25, 573-587.

56 A. W. Dobson, K. M. Erikson and M. Aschner, Ann. N. Y. Acad. Sci., 2004, 1012, 115-128.

57 S. E. Browne and M. F. Beal, Antioxid. Redox Signaling, 2006, 8, 2061-2073. 